\title{
Double-balloon enteroscopy-assisted closure of perforated duodenal diverticulum using polyglycolic acid sheets
}

Polyglycolic acid (PGA) sheets and fibrin glue adhere firmly and for a long time, are naturally absorbable, and are used in various fields, including endoscopic therapy [ $1-8]$. We report a case of perforation of a duodenal diverticulum that was treated with an endoscopic tissue-shielding method using PGA sheets and fibrin glue. A 58-year-old man was hospitalized in the nephrology department of our hospital for exacerbation of chronic renal failure due to lupus nephritis. He developed a sudden onset of severe abdominal and back pain, and an abdominal computed tomography (CT) scan showed free air in the retroperitoneum. We diagnosed a perforated diverticulum of the third portion of the duodenum.

We used an elemental diet tube to reduce the gastrointestinal (GI) pressure without surgery. However, leakage of contrast medium into the retroperitoneum was demonstrated by upper GI tract radiography ( $\bullet$ Fig. 1). We decided that it would not be possible to conservatively treat the duodenal perforation and therefore opted to perform endoscopic tissue shielding using PGA (Neoveil; Gunze, Kyoto, Japan) sheets and fibrin glue (Bolheal; Kaketsuken, Kumamoto, Japan).

We used double-balloon endoscopy (EC-450BI; Fujifilm, Tokyo, Japan) to approach the diverticulum in the third portion of the duodenum. A $100 \times 100-\mathrm{mm}$ PGA sheet was cut into $20 \times 10-\mathrm{mm}$ pieces, which were held with a biopsy forceps and delivered to the duodenal diverticulum through the channel of the scope. Once the lesion had been covered sufficiently

\section{Video 1}

A perforated duodenal diverticulum being treated by endoscopic tissue shielding using polyglycolic acid (PGA) sheets and fibrin glue.

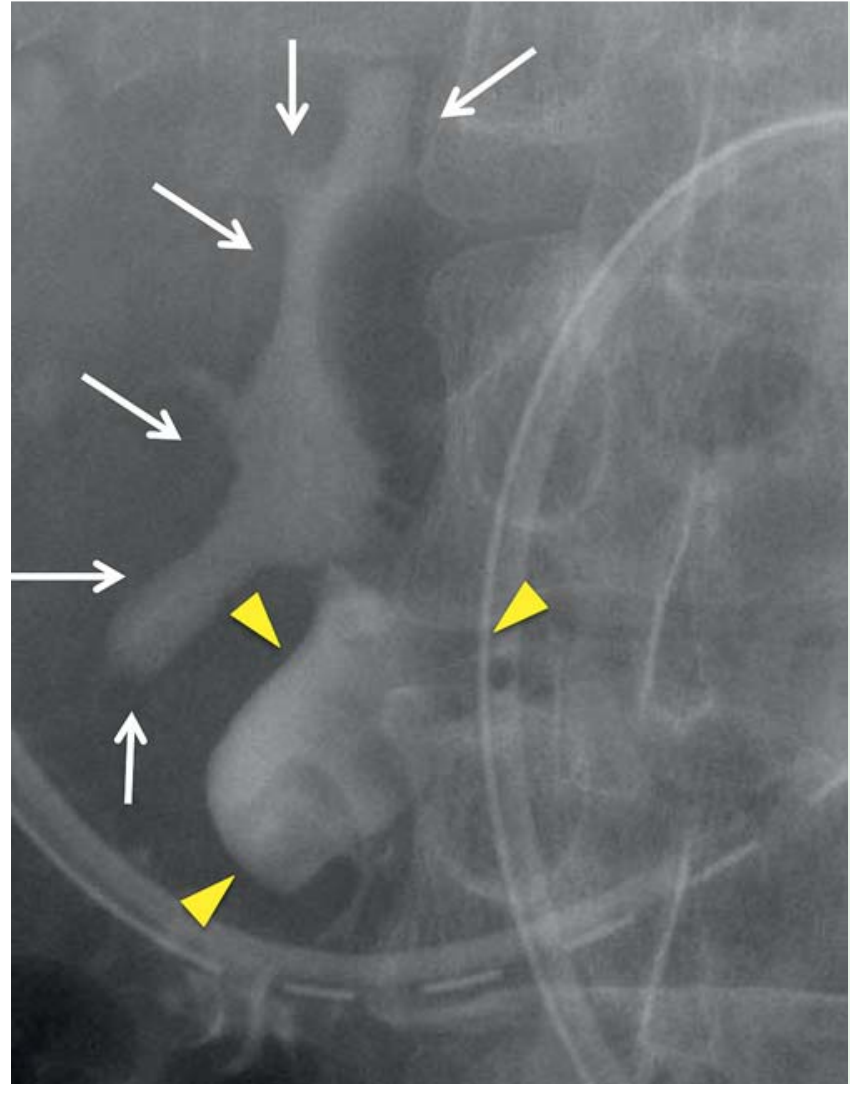

Fig. 1 Gastrointestinal tract radiography showing the duodenal diverticulum (yellow arrowheads) and leakage of contrast medium (white arrows) from the ED tube into the retroperitoneum.

with several PGA sheets, the fibrin sealant Bolheal was applied. First, $3 \mathrm{~mL}$ of solution A (fibrinogen) was applied using a catheter (PR-104Q; Olympus, Tokyo, Japan), then the same type of catheter was used to spray $3 \mathrm{~mL}$ of solution $\mathrm{B}$ (thrombin) onto the sheets to fix them to the duodenal diverticulum ( $\bullet$ Fig. 2; $\bullet$ Video 1 ).

Leakage of contrast medium into the retroperitoneum disappeared after the endoscopic therapy ( Fig.3). This method may be useful for diverticular perforation of the GI tract.

Endoscopy_UCTN_Code_TTT_1AQ_2AG

Fumisato Sasaki, Shuji Kanmura, Yuichiro Nasu, Shinichi Hashimoto, Hiroki Taguchi, Masatsgu Numata, Akio Ido

Digestive and Lifestyle Diseases, Kagoshima University Graduate School of Medical and Dental Sciences, Kagoshima, Japan

Competing interests: None 

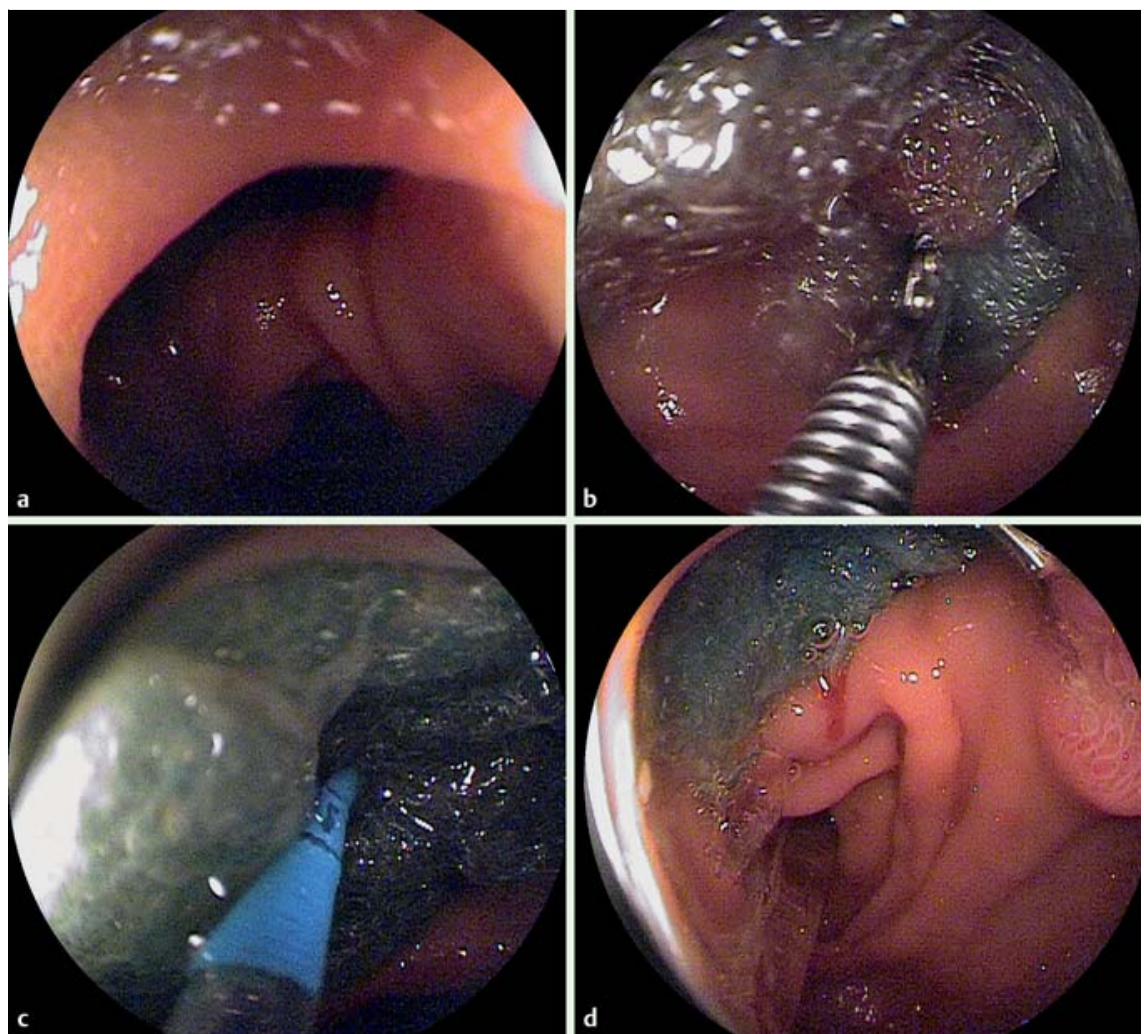

Fig. 2 Endoscopic images showing: a the opening of the duodenal diverticulum; $\mathbf{b}$ a $20 \times 10-\mathrm{mm}$ piece of polyglycolic acid (PGA) sheet being delivered to the duodenal diverticulum by biopsy forceps; c solution A (fibrinogen) of the fibrin glue solution being applied using a spray tube pressed against the sheets; $\mathbf{d}$ the PGA sheets fixed to the duodenal diverticulum by the fibrin glue solution.

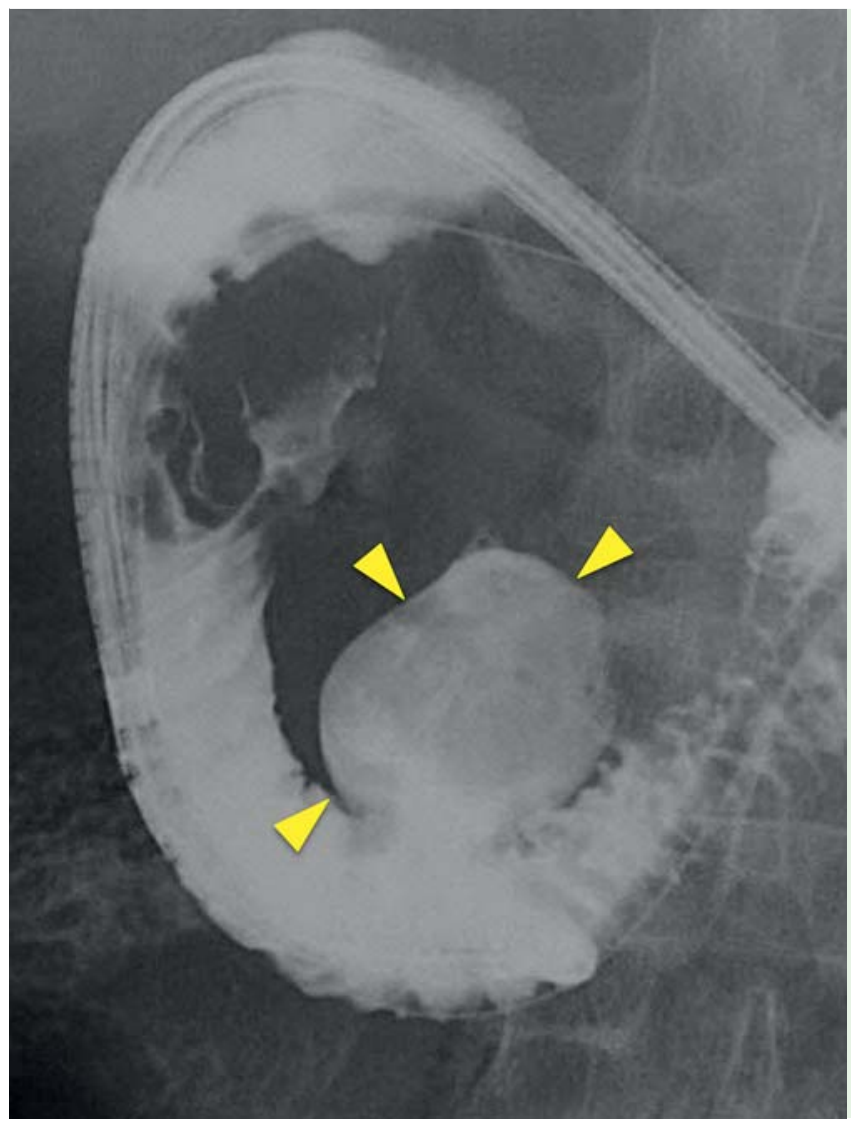

\section{References}

1 Gika M, Kawamura M, Izumi $Y$ et al. The short-term efficacy of fibrin glue combined with absorptive sheet material in visceral pleural defect repair. Interact Cardiovasc and Thorac Surg 2007; 6: $12-15$

2 Shinozaki T, Hayashi R, Ebihara $M$ et al. Mucosal defect repair with a polyglycolic acid sheet. Jpn J Clin Oncol 2013; 43: 33-36

3 Takeuchi J, Suzuki H, Murata M et al. Clinical evaluation of application of polyglycolic acid sheet and fibrin glue spray for partial glossectomy. J Oral Maxillofac Surg 2013; 71: $126-131$

4 Ueda K, Tanaka T, Hayashi $M$ et al. Meshbased pneumostasis contributes to preserving gas exchange capacity and promoting rehabilitation after lung resection. J Surg Res 2011; 167: $71-75$

5 Uemura K, Murakami Y, Hayashidani Y et al. Combination of polyglycolic acid felt and fibrin glue for prevention of pancreatic fistula following pancreatoduodenectomy. Hepatogastroenterology 2009; 56: 1538 - 1541

6 Itano $H$. The optimal technique for combined application of fibrin sealant and bioabsorbable felt against alveolar air leakage. Eur J Cardiothorac Surg 2008; 33: 457-460

7 Middleton JC, Tipton AJ. Synthetic biodegradable polymers as orthopedic devices. Biomaterials 2000; 21: 2335-2346

8 Takimoto K, Imai Y, Matsuyama K. Endoscopic tissue shielding method with polyglycolic acid sheets and fibrin glue to prevent delayed perforation after duodenal endoscopic submucosal dissection. Dig Endosc 2014; 26: 46-49

Fig. 3 Gastrointestinal tract radiography 3 weeks later showing that there was no further leakage of contrast medium into the retroperitoneum from the duodenal diverticulum (yellow arrowheads).

\section{Bibliography}

DOI http://dx.doi.org/

10.1055/s-0034-1391651

Endoscopy 2015; 47: E204-E205

(c) Georg Thieme Verlag KG

Stuttgart · New York

ISSN 0013-726X

\section{Corresponding author}

\section{Fumisato Sasaki}

Kagoshima University Graduate School of Medical and Dental Sciences

Digestive and Lifestyle Diseases

Department of Human and Environmental Sciences

8-35-1 Sakuragaoka

Kagoshima

Kagoshima-shi 890-8544

Japan

Fax: +81-99-2643504

bungohs@m2.kufm.kagoshima-u.ac.jp 